\title{
An Integrated Bioinformatics and Computational Biology Approach Identifies New BH3-Only Protein Candidates
}

\author{
Robert G. Hawley ${ }^{*}$, , Yuzhong Chen ${ }^{\dagger, 2}$, Irene Riz ${ }^{1}$ and Chen Zeng ${ }^{2}$ \\ ${ }^{I}$ Department of Anatomy and Regenerative Biology, The George Washington University, Washington, DC 20037, USA \\ ${ }^{2}$ Department of Physics, The George Washington University, Washington, DC 20052, USA
}

\begin{abstract}
In this study, we utilized an integrated bioinformatics and computational biology approach in search of new BH3-only proteins belonging to the BCL2 family of apoptotic regulators. The BH3 (BCL2 homology 3) domain mediates specific binding interactions among various BCL2 family members. It is composed of an amphipathic $\alpha$-helical region of approximately 13 residues that has only a few amino acids that are highly conserved across all members. Using a generalized motif, we performed a genome-wide search for novel BH3-containing proteins in the NCBI Consensus Coding Sequence (CCDS) database. In addition to known pro-apoptotic BH3-only proteins, 197 proteins were recovered that satisfied the search criteria. These were categorized according to $\alpha$-helical content and predictive binding to BCL-xL (encoded by BCL2L1) and MCL-1, two representative anti-apoptotic BCL2 family members, using position-specific scoring matrix models. Notably, the list is enriched for proteins associated with autophagy as well as a broad spectrum of cellular stress responses such as endoplasmic reticulum stress, oxidative stress, antiviral defense, and the DNA damage response. Several potential novel BH3-containing proteins are highlighted. In particular, the analysis strongly suggests that the apoptosis inhibitor and DNA damage response regulator, AVEN, which was originally isolated as a BCL-xLinteracting protein, is a functional BH3-only protein representing a distinct subclass of BCL2 family members.
\end{abstract}

Keywords: Apoptosis, autophagy, DNA damage checkpoint.

\section{INTRODUCTION}

Members of the B-cell lymphoma-2 (BCL2) family of proteins are characterized by conserved regions known as BCL2 homology (BH) domains (designated $\mathrm{BH} 1, \mathrm{BH} 2, \mathrm{BH} 3$ and $\mathrm{BH} 4)$. Multiple $\mathrm{BH}$ domain-containing proteins include the anti-apoptotic proteins BCL2, BCL-xL, BCL-w, MCL-1 and A1/BFL-1 and the pro-apoptotic proteins BAX, BAK, BCL2L14/BCL-G and BOK. By comparison, a subclass of pro-apoptotic BCL2 family members, referred to as BH3only proteins, possess only the $\mathrm{BH} 3$ domain. Members of this subclass include BAD, BBC3/PUMA, BCL2L11/BIM, BCL2L15/BFK, BMF, BID, BIK, HRK and PMAIP1/ NOXA $[1,2]$. Structural studies have shown that the BH3 domain of BH3-only proteins binds as an amphipathic $\alpha$ helix in a hydrophobic groove of anti-apoptotic BCL2 family members [3] and there is some evidence to suggest that the affinity of a BH3 domain peptide for anti-apoptotic BCL2 proteins is influenced by the tendency of the peptide adopt the $\alpha$-helical conformation required for binding $[4,5]$.

Day and coworkers have described a generalized motif composed of 13 residues that covers approximately two heptads of the $\alpha$-helix of BH3 domains, $\Phi_{1} \Sigma X X \Phi_{2} X X \Phi_{3} \Sigma$ ' DZ $\Phi_{4} \Gamma$, where $\Phi_{1}-\Phi_{4}$ are hydrophobic residues, $\Sigma$ and $\Sigma$ ' are small residues, $\mathrm{X}$ is any amino acid, $\mathrm{D}$ is a conserved aspar-

*Address correspondence to this author at the Department of Anatomy and Regenerative Biology, George Washington University, 2300 I Street NW, Washington, DC 20037, USA; Tel: 202-994-3511; Fax: 202-994-8885; E-mail: rghawley@gwu.edu

${ }^{\dagger}$ Current address: Ira A. Fulton Schools of Engineering, Arizona State University, Tempe, AZ 85287, USA tic acid, $\mathrm{Z}$ is usually an acidic residue and $\Gamma$ is a hydrophilic residue capable of forming an intermolecular helix cap [6]. In all bona fide $\mathrm{BH} 3$-only proteins, the $\Phi_{2}$ hydrophobic residue is a leucine (L) [7]. The conserved $\mathrm{L}$ and $\mathrm{D}$ residues form key interactions with the cognate anti-apoptotic protein. $\mathrm{L}$ is buried in the protein-protein interface and packs against conserved residues provided by the anti-apoptotic protein, while the solvent-exposed $\mathrm{D}$ forms an ionic interaction with a conserved arginine in the $\mathrm{BH} 1$ domain of the anti-apoptotic protein [8]. Other binding interactions arise from the $\Phi_{1}, \Phi_{3}$ and $\Phi_{4}$ hydrophobic residues. All anti-apoptotic proteins use the equivalent hydrophobic groove interface to bind multiple BH3-only proteins but they have distinct $\mathrm{BH} 3$ binding profiles [9-11]. In this regard, introducing two or three mutations into the BIM BH3 sequence can generate considerable selectivity for BCL-xL relative to MCL-1 or vice versa [12, 13]. Recently, Dutta and colleagues combinatorially mutated all four hydrophobic residues $\left(\Phi_{1}-\Phi_{4}\right)$, the conserved $\mathrm{D}$, and a sixth position of the BIM BH3 domain ( $\mathrm{X}$ at position $3 \mathrm{~b}$ according to their numbering convention; see Fig. 1) and used yeast surface display to identify peptides selective for binding to BCL-xL or MCL-1 [11]. They complemented this approach by performing binding assays with $\mathrm{BCL}-\mathrm{xL}$ or MCL-1 and peptide arrays spotted with BIM BH3 peptides in which 10 of the 13 residues (including the 6 randomized in the yeast surface display library) were individually mutated. Using the combined data, they developed positionspecific scoring matrix (PSSM) models for BH3 binding specificity for BCL-xL and MCL-1.

Our group has a long-standing interest in the molecular mechanisms underlying growth and survival of malignant cells [14-18]. Here we describe an approach that combines 


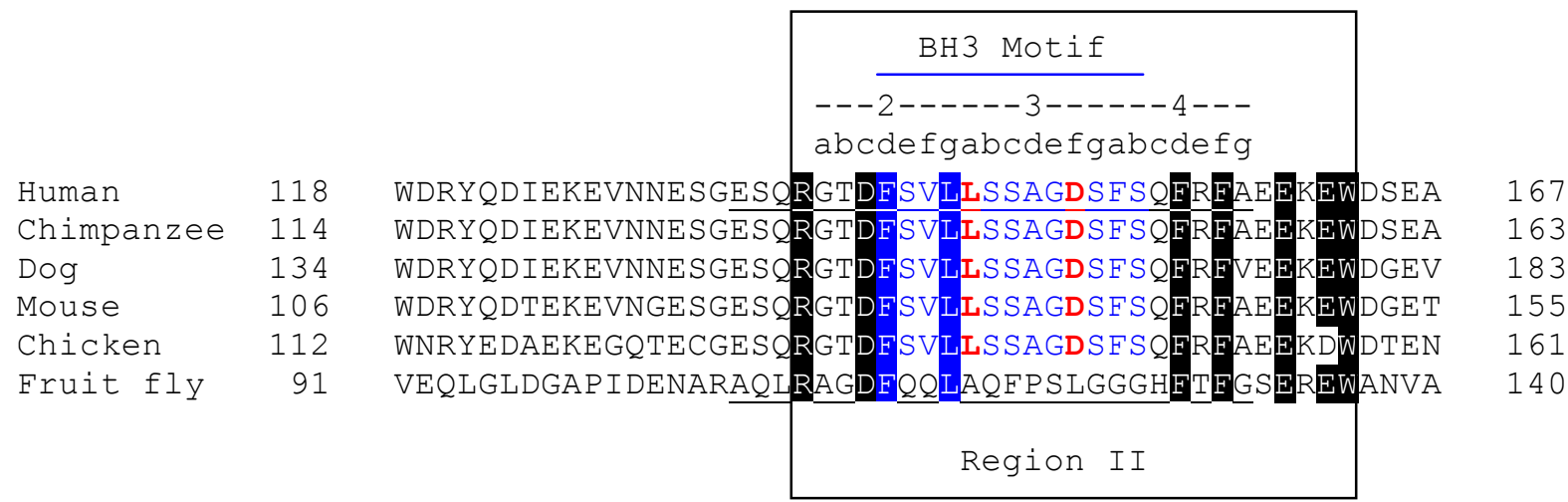

Fig. (1). Alignment of AVEN proteins of human (NP_065104.1), chimpanzee (XP_510277.3), dog (XP_535418.2), mouse (NP_083120.2), chicken (NP_001005791.1) and fruit fly (NP_572817.1). Conserved BH3-like motifs in the human, chimpanzee, dog, mouse and chicken proteins are highlighted in blue with conserved L and D residues in red. Numbering using the heptad convention of Dutta and colleagues [11] is shown at the top. Note that the Drosophila protein has diverged considerably from the other proteins and does not contain a putative BH3 motif, but some common residues have been retained within the boxed region (referred to as Region II by Zou and colleagues [34]). The 25-mer AVEN and dAven peptides used in the molecular dynamics simulations of putative BH3 interactions with BCL-xL (Fig. 3) are underlined.

genome-wide consensus BH3 motif searching, $\alpha$-helical secondary structure prediction and predictive PSSM models of binding to BCL-xL and MCL-1 in an attempt to identify novel BH3-containing proteins involved in cancer pathobiology and therapeutic response.

\section{MATERIALS AND METHODOLOGY}

\section{Bioinformatics}

A search for new BH3-containing BCL2 family members was conducted using the NCBI CCDS database (http://www. ncbi.nlm.nih.gov/projects/CCDS/CcdsBrowse.cgi) for proteins containing 13-amino acid sequences that match a consensus BH3 motif, $\Phi_{1} \Sigma X X \Phi_{2} \mathrm{XX} \Phi_{3} \Sigma$ 'DZ $\Phi_{4} \Gamma$, based on that described by Day and colleagues [6] with the following modifications: $\Phi_{1}$ represents ACGILMFPWVYET (BNIP1/ BNIP3L has E; HRK has T) [13]; $\Phi_{2}$ is L; $\Phi_{3}$ represents ACGILMFPWVYETS (BECN1 has T; BNIP3L has S) [13]; $\Phi_{4}$ represents ACGILMFPWVYET [13]; $\Sigma$ represents GASCV (BOK has C; BCL2L14/BNIP3L have V); $\Sigma$ ' represents GASC; $\mathrm{X}$ is a random amino acid; $\mathrm{D}$ is a conserved aspartic acid; $Z$ represents DEKQLSW (NOXA has K; BCL2L14/BCL2L15/BMF have Q; BBC3/BECN1 have L; BID has S; BNIP3L has $\mathrm{W}$ ); and $\Gamma$ represents an amino acid which is capable of forming an intermolecular helix cap, NDSTCGHYEV [19] .

The helical content of the putative $\mathrm{BH} 3$ sequences was determined from secondary structure predictions of all of the human protein sequences in the NCBI CCDS database using PredictProtein (www.predictprotein.org) PROFphd neural network secondary structure prediction software [20].

PSSM models derived by Dutta and colleagues were used to predict binding to BCL2L1/BCL-xL and MCL-1 [11]. The PSSM models were built using peptide array data from point mutations made in the background of a peptide derived from the $\mathrm{BH} 3$ region of BCL2L11/BIM (involving 10 positions2d, 2e, 2g, 3a, 3b, 3d, 3e, 3f, 3g, 4a-mutated to any of ADEFGHIKLNPQRSTV). The binding affinity score presented for each putative $\mathrm{BH} 3$ sequence is the sum of the score contributions of all 10 positions predicted by the revised PSSM model that incorporated 170 single-site mutations plus selected sequences based on combinatorial mutations identified by yeast surface display [11].

\section{Molecular Dynamics Simulations}

Molecular dynamics simulations of interactions between putative BH3 sequences and anti-apoptotic BCL2 family members were carried out essentially as we have described previously [21] except that GROMACS (Groningen Machine for Chemical Simulations) was used as the modeling software (www.gromacs.org) [22]. The starting coordinates for the simulations of the binding of the 25-mer AVEN and dAven peptides to BCL-xL were based on the BAD/BCL-xL complex (PDB code: 1G5J) [4]. The backbone conformation for the two peptide ligands ESQRGTDFSVLLSSAGDSF SQFRFA (AVEN) and AQLRAGDFQQLAQFPSLGGGHF TFG (dAven) was obtained from amino acids 301-325 of BAD with all sidechains rebuilt using the SCAP sidechain prediction program [23]. The GROMACS96 53a6 force field and Simple Point Charge (SPC) water model were chosen for the simulations. Using the "Pdb2gmx" tool available in GROMACS, hydrogen atoms present in the file were removed and then rebuilt according to the description in the force field. Energy minimization was performed on the initial structure of the ligand and receptor complex to relax possible strain after adding the force field and water model by using steepest-descent integration. The system was equilibrated for a period of $0.5 \mathrm{~ns}$. The complex was then placed in the center of a cubic water box and the solute molecules were solvated with SPC water molecules using the GROMACS utility program "Genbox". The total number of atoms in the two simulations varied between 26,000 and 27,000 . The system was neutralized by adding $\mathrm{Na}^{+}$or $\mathrm{Cl}^{-}$ ions to a concentration of $0.15 \mathrm{M} \mathrm{NaCl}$ using the GROMACS utility program "Genion". In some simulations, excess $\mathrm{Na}^{+}$or $\mathrm{Cl}^{-}$ions were added. This step was followed by an energy minimization and a position relaxation simulation, each for $0.5 \mathrm{~ns}$. Afterwards, the system was equilibrated for a period of $1 \mathrm{~ns}$ in two stages. First, a $0.5 \mathrm{~ns}$ stage 
simulation was performed under the NVT ensemble (constant number of atoms, volume and temperature), relaxing the system and coupling it to the heat bath. The second stage consisted of a further $0.5 \mathrm{~ns}$ simulation without any restraints using the NPT ensemble (constant number of atoms, pressure and temperature). Production runs constituted $20 \mathrm{~ns}$ of simulations under NPT conditions. The LINCS algorithm [24] was used to constrain all bonds involving hydrogen atoms. Periodic boundary conditions were applied in all three directions. The simulation temperature and pressure were $300 \mathrm{~K}$ and 1 bar, respectively. Temperature was maintained by using the Berendsen algorithm [25] with a coupling constant of 0.1 ps during the equilibration and production stages. For pressure coupling, the Berendsen algorithm with a coupling constant of $1.0 \mathrm{ps}$ was adopted to maintain the boundary pressure. Non-bonded interactions were evaluated using a spherical cutoff of $14 \AA$. A time step of $0.001 \mathrm{ps}$ was used for the simulations.

\section{RESULTS AND DISCUSSION}

\section{Bioinformatics Search for Novel BH3-Only Proteins}

By using a relaxed consensus sequence corresponding to BH3-containing pro-apoptotic BCL2 family members (see Materials and Methodology), we conducted a search of the NCBI CCDS database. A total of 279 sequences were recovered representing 214 genes (17 known $\mathrm{BH} 3$-containing genes and 197 potential $\mathrm{BH} 3$-only protein candidates) that satisfied the search criteria. As expected, included in this list were all of the known pro-apoptotic BH3-only proteins (BAD, BBC3/PUMA, BCL2L11/BIM, BCL2L15/BFK, BMF, BID, BIK, HRK, and PMAIP1/NOXA). Also present in the list were the multidomain pro-apoptotic proteins $\mathrm{BAK}$, BAX, BCL2L14/BCL-G and BOK. In addition, two BH3containing proteins involved in autophagy, BECN1 and BNIP3L/NIX, were identified as were the previously recognized BH3-containing proteins HUWE1/MULE, an E3 ligase that ubiquitinates MCL-1, and RAD9A, a multifunc- tional protein involved in cell death and the DNA damage checkpoint (Supplemental Files 1 and 2).

A PSSM was used to predict binding to BCL2L1/BCL$\mathrm{xL}$ and MCL-1. The PSSM was derived by Dutta and colleagues from binding analyses using peptide arrays and yeast surface display in which the effects of single-site and combinatorial point mutations were assessed in the background of a peptide corresponding to the BH3 region of BCL2L11/ BIM [11]. A prediction could be made for 111 of the 197 candidates, and these are listed in Supplemental File 2 (pages $6-45)$ in the descending order of their binding affinity scores for BCL2L1/BCL-xL. For the remaining candidates (86 genes), a prediction was not possible because at least one of the amino acids in the motif was not contained in the PSSM matrix (Supplemental File 2, pages 46-72). Below we highlight several of these proteins as potential new $\mathrm{BH} 3-$ containing BCL2 family members.

\section{Putative BH3-Containing BCL2 Family-Binding Proteins}

\section{AVEN (Apoptosis, Caspase Activation Inhibitor)}

AVEN (also known as PDCD12; programmed cell death 12) is a novel apoptosis inhibitor which binds to both BCL$\mathrm{xL}$ and the caspase regulator, apoptotic peptidase-activating factor-1 (Apaf-1) [26]. AVEN has been proposed to inhibit apoptosis in part by stabilizing BCL-xL [27]. Apaf-1 is a pro-apoptotic protein that accelerates apoptosis downstream of mitochondria by multimerizing to form the apoptosome complex, which activates caspase proteases [28]. AVEN interferes with the ability of Apaf-1 to self-associate, suggesting that AVEN also impairs Apaf-1-mediated activation of caspases [26].

AVEN was identified in a yeast two-hybrid screen with BCL-xL as bait; the gene is broadly expressed and is conserved in other mammalian species. Indirect immunofluorescence analysis indicated a diffuse nuclear staining pattern and a reticular cytoplasmic staining pattern consistent with intracellular membrane localization [26]. We identified a

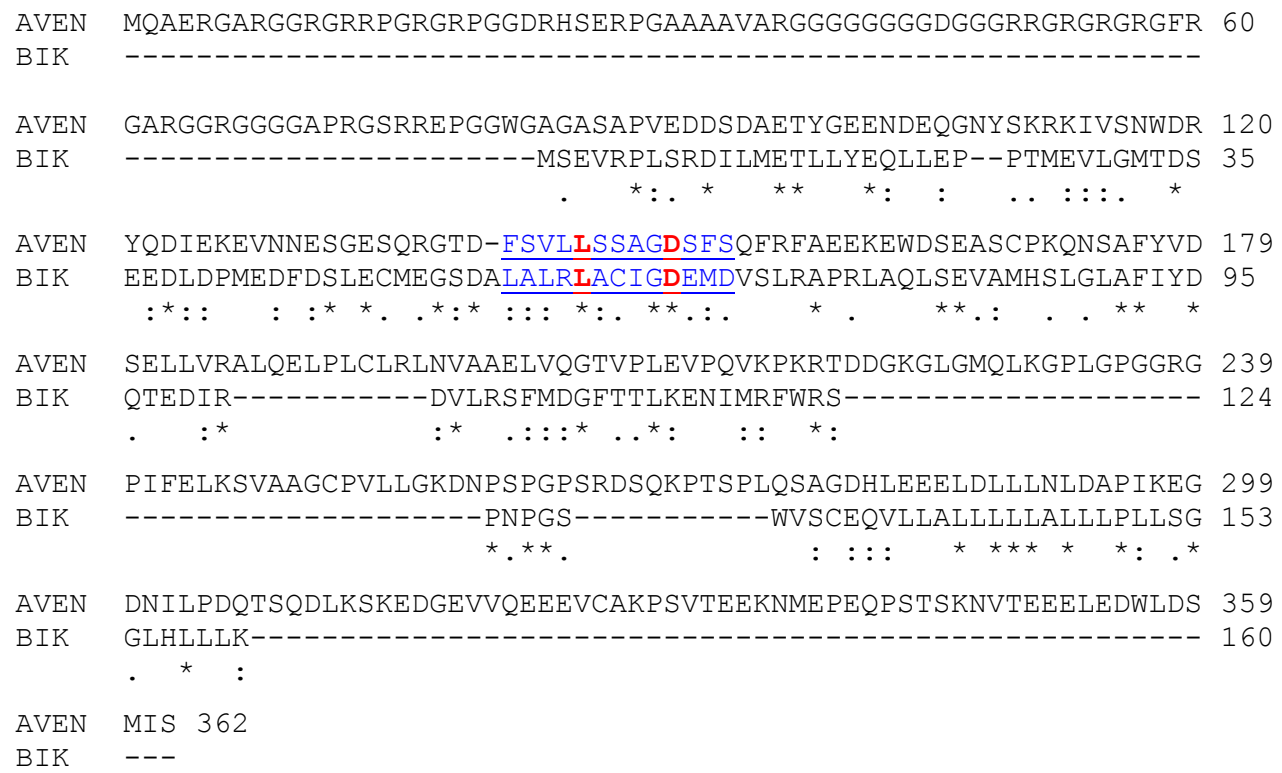

Fig. (2). ClustalW2 (http://www.ebi.ac.uk/Tools/msa/clustalw2/) sequence alignment of AVEN versus BIK. “*”, identical; “:”, conserved substitutions; ".”, semi-conserved substitutions. The putative BH3-like motif in AVEN and the BIK BH3 domain are highlighted. 
putative $\mathrm{BH} 3$ motif in AVEN encompassing amino acids 141-153 (FSVLLSSAGDSFS; Fig. 1). Although BH3-only proteins share homology mainly in the $\mathrm{BH} 3$ region $[1,29]$, it is noteworthy that further sequence similarity can be found between AVEN and BIK [30] outside of the BH3 motif (58\% similarity over a 77 amino acid region encompassing the putative $\mathrm{BH} 3$ motif (Fig. 2). The putative AVEN BH3 motif contains two amino acids that are associated with BCL-xL specificity (FSVLLSSAGGSFS) according to binding studies carried out by Dutta and colleagues [11]. According to the PSSM model, AVEN would be predicted to bind BCL-xL with higher affinity than BCL2L14/BCLG [31] (see Supplemental File 2).

Chau and coworkers reported that AVEN binding to BCL-xL involved both the BH1 and BH4 domains of BCL$\mathrm{xL}$ [26]. The $\mathrm{BH} 1$ domain is part of the hydrophobic groove that binds $\mathrm{BH} 3$ motifs [32], suggesting a $\mathrm{BH} 3$-mediated interaction. N136 of BCL-xL forms a hydrogen bond with a carboxylate group of the conserved D in the BH3 motif [32]. Mutation of N136 to I eliminates this and leads to loss of a stabilizing interaction. One of the BCL-xL mutants that Chau and colleagues used that disrupted the BCL-xL-AVEN interaction (BCL-xL mutant 7) contained the N136I mutation (V135A/N136I/W137L). Additionally, because AVEN bound to a deletion mutant of BCL-xL encompassing amino acids $26-83$ but not to an N-terminally truncated mutant missing the first 61 amino acids, the $\mathrm{N}$-terminal 25 amino acids of BCL-xL were implicated in the interaction. The Nterminal 25 amino acids of BCL-xL comprise the BH4 domain, suggesting a potential interdependency of the BCL$x L-A V E N$ interaction interfaces similar to that described between BCL2 and BAX [33]. Consistent with the above speculation, the putative $\mathrm{BH} 3$ motif is conserved in Aven molecules across species (referred to as Region II in Fig. 1 of Ref. [34]). The exception to this is the Drosophila ortholog of Aven (dAven) that was recently identified by Zou and coworkers [34]. Interestingly, the Drosophila protein does not contain a recognizable $\mathrm{BH} 3$ motif: the conserved $\mathrm{L}$ and $D$ residues as well as other amino acids are not preserved (e.g., an $\mathrm{F}$ at position 4a that AVEN shares with BIM and BAD and which makes a significant hydrophobic contribution to the $\mathrm{BH} 3$ domain interactions of these proteins; Supplemental File 1) (Fig. 1).

Recent molecular dynamics simulations of pro-apoptotic BH3 peptide helices in aqueous medium have suggested a relationship between helix stability and their binding affinities to BCL-xL. Lama and Sankararamakrishnan speculated that unlike the situation when the $\mathrm{BH} 3$ domains are part of a polypeptide chain and the hydrophobic residues insert themselves into the hydrophobic groove of an anti-apoptotic BCL2 family member, small peptides exposed to aqueous medium are likely to cluster among themselves to minimize contact with water molecules [5]. Therefore, the peptide simulations conducted represented an unfolding study and the authors were able to correlate the helical state of the $\mathrm{BH} 3$ peptide at the end of the simulation with its experimental binding affinity for BCL-xL [4,5]. The stable helical segment that was found to be common to all the $\mathrm{BH} 3$ peptides is composed of a short five residue region with the conserved $\mathrm{L}$ located in the center, and they speculated that the conserved $\mathrm{L}$ could serve as a nucleation signal for an eventual longer helix upon binding to BCL-xL. We carried out molecular dynamics simulations of BCL-xL in complex with a 25-mer corresponding to the putative $\mathrm{BH} 3$ motif of AVEN and the corresponding region of dAven (highlighted in Fig. 1) [35]. As can be seen in Fig. 3, whereas the AVEN peptide exhibited significant internal helical stability at the end of the simulation period (Fig. 3A), there was almost a complete loss of helical character of the dAven peptide (Fig. 3B). Based on Lama and Sankararamakrishnan's work [5,35], dAven would be predicted to have significantly reduced (if any) binding affinity for BCL-xL. Experimental data supports this prediction. In contrast to AVEN, no interaction was found between dAven and Drosophila BCL2 family members [34].
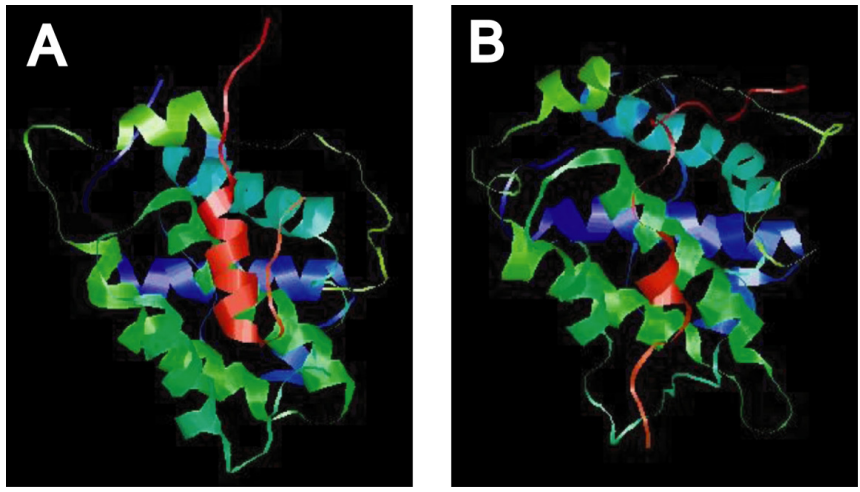

Fig. (3). Results of molecular dynamics simulation of putative AVEN BH3 motif with BCL-xL. (A). Ribbon diagram of a peptide encompassing the putative BH3 motif of human AVEN (ESQRGTDFSVLLSSAGDSFSQFRFA; shown in red) complexed with BCL-xL. (B) Ribbon diagram of the corresponding peptide from Drosophila Aven (AQLRAGDFQQLAQFPSLGGGHFTFG; shown in red). See Fig. (1) and text for details. Simulations (20 ns) are based on an analogous 25-mer of the BAD BH3 motif and the $\mathrm{BAD} / \mathrm{BCL}-\mathrm{xL}$ structure (PDB code: 1G5J).

Collectively, these enticing correlations raise the strong possibility that the $\mathrm{BH} 3$ motif in AVEN is a functional $\mathrm{BH} 3$ domain and that AVEN is a novel $\mathrm{BH} 3$-only protein. In addition to anti-apoptotic activity, AVEN also exhibits cell cycle regulatory function. After DNA damage, AVEN induces cell-cycle arrest via ataxia-telangiectasia-mutated (ATM) kinase activation [36]. A working hypothesis is that human AVEN (and most Aven orthologs) is a multifunctional protein that modulates decisions of cell-cycle arrest and apoptosis during the DNA damage response, depending on the extent of damage [37]. In this regard, AVEN exhibits some striking functional similarities with RAD9A [38-40]; additional analogies can be drawn between AVEN and BID $[41,42]$.

\section{GRAMD4}

Proteins containing GRAM (Glucosyltransferases, Rablike GTPase activators and Myotubularins) domains are predominately involved in membrane-associated processes [43]. GRAMD4 was originally identified as a downstream pro-apoptotic target of the transcription factor E2F1 (termed Death-Inducing-Protein), which localizes to mitochondria [44]. Upregulation of endogenous GRAMD4 by E2F1 activation or GRAMD4 overexpression demonstrated greatly reduced cell viability in several in vitro systems accompanied by typical apoptotic features such as caspase-3 
activation and cleavage of poly(ADP-ribose)-polymerase. Inhibition of endogenous protein function by small interfering RNA rescued p53-negative cells from E2F1-induced apoptosis, indicating that GRAMD4 is an essential mediator of the p53-independent E2F1 death pathway.

Recent studies by the same group have provided evidence that GRAMD4 is a p73 primary-response gene [45]. Pützer and colleagues showed that GRAMD4 is highly efficient in inducing mitochondrial outer membrane permeabilization with release of the intermembrane space proteins cytochrome c and Smac/Diablo into the cytoplasm. Overexpression of $\mathrm{p} 73 \alpha$ and $\mathrm{p} 73 \beta$ isoforms, but not $\mathrm{p} 53$, led to direct GRAMD4 promoter transactivation. GRAMD4 was found to physically interact with BCL2, promoting BAX mitochondrial relocalization and oligomerization. In addition, GRAMD4 induced changes in BCL2 and BAX protein levels independent of transactivation effects at the level of the BCL2 or BAX promoters, suggesting that GRAMD4 is functioning through indirect perhaps post translational mechanisms, consistent with its apparent lack of a DNA binding and transactivation domain [44]

Our analysis identified a putative $\mathrm{BH} 3$ motif in GRAMD4 encompassing amino acids 218-230 (FVKNL SALSDWYS). It is noteworthy that, with the exception of $\mathrm{BECN} 1$, in all of the bona fide $\mathrm{BH} 3$-only genes reported to date, the $\mathrm{BH} 3$ domain is a structural and functional subunit encoded by a single short exon [29]. As shown in Table 1, the putative $\mathrm{BH} 3$ motif in GRAMD4 satisfies this condition. Although the authors did not detect a BH3 domain upon inspection of the protein, they reported that deletion of a region encompassing amino acids 92-176 disrupted the interaction of GRAMD4 with endogenous and ectopically expressed BCL2 protein. It remains to be demonstrated, but a possibility is that the 92-176 amino acid deletion in GRAMD4 led to a conformational change in the putative $\mathrm{BH} 3$ domain disrupting the interaction with the $\mathrm{BH} 1-\mathrm{BH} 3$ hydrophobic groove interface of BCL2 $[33,46]$.

\section{HIP1R}

HIP1R (Huntingtin interacting protein 1 related) is a multi-domain protein that regulates clathrin-mediated endocytosis and actin assembly [47]. The related HIP1 protein was identified as an interactor of huntingtin, a protein that when mutated is involved in the genetic neurodegenerative disorder Huntington's disease $[48,49]$. The mutated huntingtin has reduced affinity for HIP1, suggesting that the impaired interaction could be part of the disease mechanism [49]. HIP1R was identified on the basis of structural homology to HIP1 [50,51]. Both proteins have an $\mathrm{N}$ terminally localized ANTH (AP180 N-terminal homology) domain, a central coiled-coil domain, and a talin-like domain at the C-terminus. HIP1R binds inositol lipids via the ANTH domain [52] and actin via its talin like-domain [53]. In contrast to HIP1, HIP1R does not directly bind huntingtin [54].

HIP1 was previously shown to have transforming function due to alterations in receptor trafficking [55]. Other work studying ANTH domains, suggested a mechanism by which the protein might facilitate cell survival by stabilizing receptor tyrosine kinases by binding 3-phosphorylated inositol lipids [56]. Hyun and coworkers provided evidence in support of such a mechanism for both HIP1 and HIP1R [52]. The authors speculated that HIP1 and HIP1R might

Table 1. BH3 Motifs Encoded by a Single Exon

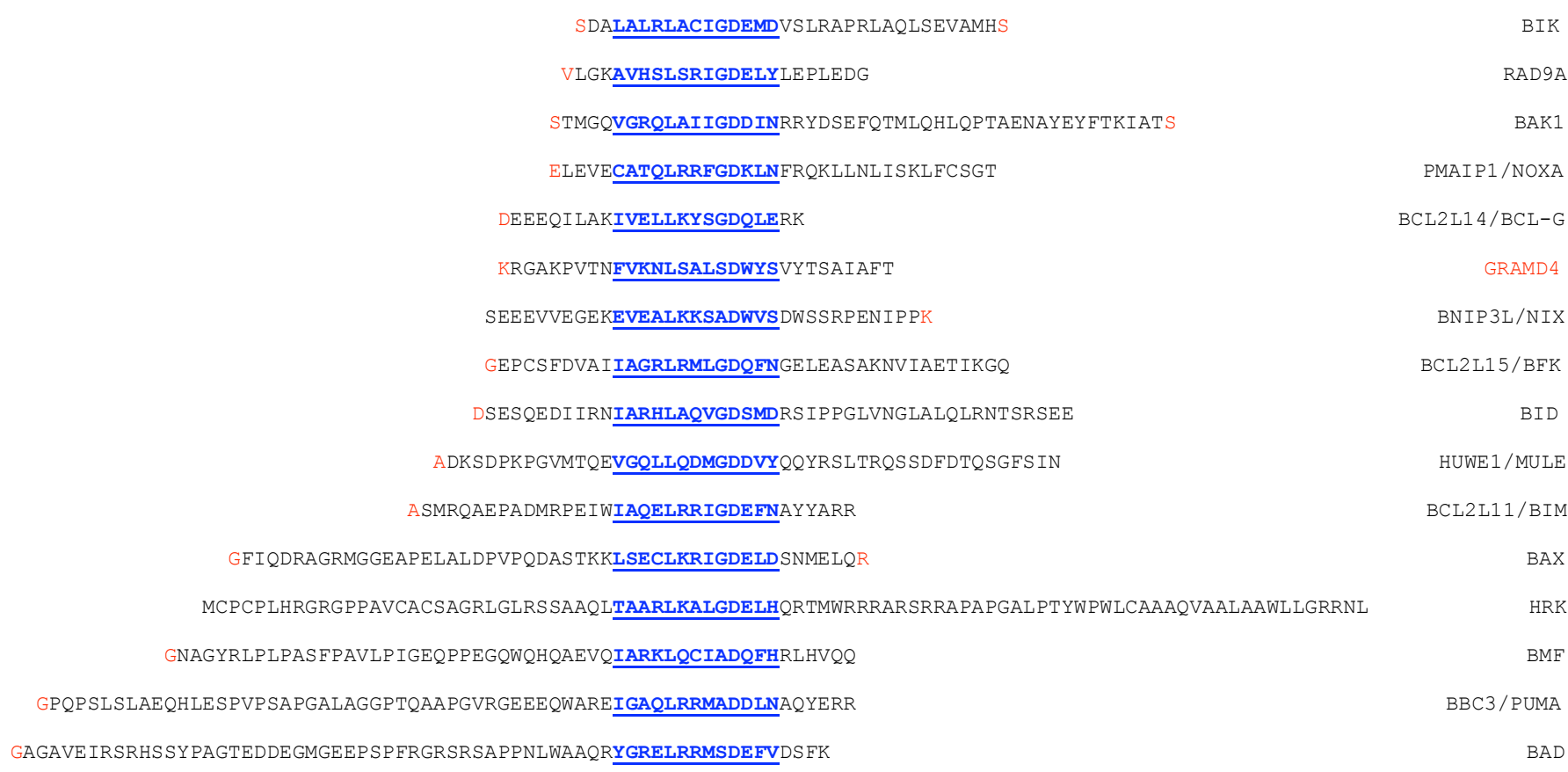

With the exception of BECN1 (see Table 2), the BH3 motif in BH3-only BCL2 family proteins is encoded by a single short exon [29]. Known and putative $\mathrm{BH} 3$ motifs are in underlined bold blue type.

Red highlighting indicates an amino acid encoded across a splice junction.

Amino acid sequences are from the CCDS dataset (http://www.ncbi.nlm.nih.gov/projects/CCDS/CcdsBrowse.cgi). 
Table 2. BH3 Motifs Encoded by Two Exons

RMMS TESANSETLI GEAS DGGTMENLSRRLKVTGDLFDIMSGQT DVDHPLCEECTDTLLDQLDTQLNVTENECQNYK

GAATQQEFYAKNSRWTEGLISASKAVGWGATQLVEAADKVVLHTGKYEELIVCSHEIAASTAQLVAASK

VWQGLDVVRTSRAL IGATNPADAP PGTIRGDFCIEVGKNLIHGSDSVESARREIALWFRADELLCWEDSAGHWLYE

VEDDSDAETYGEENDEQGNYSKRKIVSNWDRYQDIEKEVNNESGESQRGTDFSVLLSSAGDSFSQFRFAEEKEWDSEASCPKQ

EMPDSAAALLGTCCKLQI IPTLCHLLRALSDDGVSDLEDPTLTPLKDTERFGIVQRLFASADISLERLKSSVKAVI LKDSKVFPLLLCITLNGLCALGREHS

MEVLRRSSVFAAEIMDAFDRSPTDKELVAQAKALGREYVHARLLRAGLSWSAPERAAPVPGRLAEVCAVLLRLGDELEMIRPSVYRNVAROLHISLOSEPVVTDAFLAVAGH IFSAG
BECN1

HIP1R

NME3

AVEN

DENA 5

Prior to the discovery of BECN1, BOK (a multidomain pro-apoptotic protein) was the only BCL2 family member in which the BH3 motif was found to be encoded by two exons [29].

Known and putative BH3 motifs are in underlined bold type.

Blue highlighting indicates a different exon.

Red highlighting indicates an amino acid encoded across a splice junction.

Amino acid sequences are from the CCDS dataset (http://www.ncbi.nlm.nih.gov/projects/CCDS/CcdsBrowse.cgi).

bind to inositol lipid determinants of clathrin-coated vesicles, early endosomes, and recycling endosomes via their ANTH domains, causing the endocytic machinery to favor continued signaling either by stabilizing the early endosome or by stimulating growth factor receptor recycling to the cell surface.

Conflicting data exists as to whether overexpression of HIP1 has pro-apoptotic $[57,58]$ or anti-apoptotic functions [59]. In particular, Rao and colleagues found that expression of an ANTH deletion mutant of HIP1, but not full-length HIP1, induced cell death in a dominant interfering manner [59]. The authors noted that two other reports of proapoptotic activity used a different HIP1 cDNA which lacked the N-terminal sequence encoded by an upstream exon that had not been previously appreciated [60]. Thus, they suggested that the reason for the discrepancy may be that cDNA used in the previous studies was functionally similar to the ANTH deletion mutant of HIP1 that they used. Following up on the HIP1 findings, Hyun and coworkers reported that a HIP1R mutant lacking the ANTH domain but not full-length HIP1R similarly induced apoptosis after ectopic expression in HEK293T cells [52].

Recently, HIP1R was identified in a screen for interacting partners of BCL2L10 (human DIVA or BCL-B), a member of the BCL2 family that can either function as a pro- or anti-apoptotic protein [61]. Under the conditions employed in that study, ectopic expression of HIP1R induced apoptosis and augmented BCL2L10 association with caspase 9 . In addition, a member of the proapoptotic BCL2 family, BAK, was required for HIP1R to induce cell death, while BAX was dispensable. Deletion of both the N-terminal ANTH domain and the C-terminal talin-like domain of HIP1R greatly compromised its binding ability to BCL2L10. The C-terminal deletion (amino acids 816-1068) encompassed a putative BH3 motif (amino acids 882-894) that we identified (GATQLVEAADKVV; Table 2).

In addition to its well-accepted role as an antagonist of apoptosis, BCL2 has also been reported to function as a regulator of cell motility by interacting with and enhancing actin polymerization $[62,63]$. Besides binding to actin [47], it is evocative that HIP1R was identified in a proteomic screen for proteins that interact with dynein light chain LC8 (although incorrectly listed as HIP1 in Table 2 of Ref. [64]). LC8 is a component of the dynein motor complexes that selectively sequester the the $\mathrm{BIM}_{\mathrm{L}}$ and $\mathrm{BIM}_{\mathrm{EL}}$ isoforms of $\mathrm{BIM}$ and so regulate their cell death-inducing functions [6567]. In response to cellular damage that affects the cell cytoskeleton, BIM-LC8 complexes are released into the cytoplasm where they can interact with anti-apoptotic BCL2 proteins, via their $\mathrm{BH} 3$ domains. These latter observations suggest further parallels between HIP1R and typical BH3only proteins.

\section{Putative BH3-Containing Proteins Associated with Apoptosis}

\section{NME3}

NME3 (non-metastatic cells 3) is a member of a family of 10 genes in humans named after the prototypic member NME1, which was identified as a suppressor of tumor metastasis (Nm23-H1) and exhibits nucleoside diphosphate kinase activity $[68,69]$. NME proteins are involved in a variety of physiological and pathological cellular processes including cell proliferation, differentiation, vesicular trafficking and adhesion as well as apoptosis [70]. Phylogenetic analysis of NME proteins shows two strongly supported distinct clusters, with NME3 belonging to same group as NME1, NME2 and NME4 [71].

NME3 was first identified as a gene associated with the blast crisis phase of chronic myeloid leukemia [72]. A characteristic feature of blast crisis is the differentiation arrest of the malignant hematopoietic progenitor cells. Constitutive expression of NME3 in a myeloid precursor cell line, which is growth-factor dependent for both proliferation and differentiation, resulted in inhibition of differentiation. Notably, in the cell line model, differentiation arrest was associated with apoptotic cell death [72]. The mechanisms responsible for differentiation arrest and induction of apoptosis in this system are not known. A role for NME3 in the regulation of differentiation and apoptosis was also reported in neuroblastoma cells. However, in this situation overexpression of NME3 promoted differentiation while protecting the cells from apoptosis induced by serum deprivation [73]. The effects of NME3 expression thus seem to be dependent on cellular context.

We identified a putative BH3 motif in NME3 encompassing amino acids 129-141 (VGKNLIHGSDSVE; Table 2). That NME3 might associate with BCL2 family members 
is suggested from studies with the related human and rat NME2 proteins. NME2 was identified in a screen for proteins that interact with BCL2L10, a member of the BCL2 family that can either function as a pro- or anti-apoptotic protein, described above [74]. BCL2L10 resides in the membranes of intracellular organelles [75] and can translocate from endoplasmic reticulum to mitochondria upon apoptotic stimulus [76]. Kang and colleagues showed that ectopic expression of NME2 in HEK293T cells induced apoptosis where it colocalized with BCL2L10 in the cytoplasm. NME3 has been reported to be localized within the cytoplasm and, at least partly, within mitochondria although no specific mitochondrial targeting sequence has been found [73,77]. NME2 contains a variant of the putative $\mathrm{BH} 3$ motif found in NME3 (VGRNIIHGSDSVE) encompassing amino acids 112-124) which would satisfy a broader consensus definition but was not identified in our screen because of a conservative substitution of the $\Phi_{2}$ hydrophobic L with isoleucine.

\section{DFNA5}

The DFNA5 (deafness, autosomal dominant 5) gene was first identified as a gene whose expression is inversely correlated with that of the estrogen receptor in breast carcinomas (accordingly, it was initially designated as ICERE-1 for inversely correlated with estrogen receptor expression) [78] and independently as a gene causing autosomal dominant hearing loss [79]. Epigenetic silencing of DFNA5 in several types of cancer, including a large percentage of gastric, colorectal and breast cancers, have implicated DFNA5 as a tumor suppressor gene [80-82].

In one study, decreased expression of DFNA5 in melanoma cell lines correlated with increased resistance to etoposide-induced apoptosis [83]. Subsequent work identified DFNA5 as a p53 target gene and its ectopic expression enhanced etoposide-induced cell death [84]. Intriguingly, in the same study, ectopic expression of DFNA5 in TP53-negative lung cancer cells inhibited etoposide-induced cell death. These contrasting results suggested that DFNA5 plays a role in the p53-regulated cellular response to genotoxic stress by somehow cooperating with $\mathrm{p} 53$, although the function of DFNA5 in this process remains to be elucidated. The DFNA5 gene has also be reported to be a glucocorticoidinducible gene in $\mathrm{T}$ cell acute lymphoblastic leukemia cells. Induction of DFNA5 mRNA correlates with glucocorticoiddependent apoptosis of the cells. In this case, however, its expression alone was not sufficient for apoptosis induction [85].

A potential explanation for the differing results and insight into the mechanism of DFNA5-induced hearing loss was provided by identification of a fifth family with the disorder [86]. Comparative analyses of the five mutant DFNA5 genes revealed different mutations but in each case the DFNA5 mRNA transcript was found to skip exon 8, resulting in a frameshift and a premature truncation of the protein. These findings suggested that DFNA5-associated hearing loss is attributable to a highly specific gain-of-function mutation, in which skipping of one exon causes disease. Experimental evidence congruent with this hypothesis was previously provided by the finding that transfection of mutant DFNA5 causes cell death [87]. Following up on these observations, de Beeck and coworkers recently demonstrated that DFNA5 is composed of two domains, separated by a hinge region. The first region (exon 2-exon 7) induces apoptosis when transfected in HEK293T cells, leading to the speculation that the second region may mask this domain and regulate its apoptosis inducing capability [88]. The DFNA5 exon 2-exon 7 construct was expressed mainly at the plasma membrane. In addition, it was observed in the cytoplasm in a granulated manner. The putative $\mathrm{BH} 3$ motif in DFNA5 is formed across the splice junction of exons 9 and 10 (LCHLLRALSDDGV; Table 2). Therefore, a potential scenario is that the $\mathrm{BH} 3$-like domain encoded by exons 9 and 10 of DFNA5 facilitates association of the protein with BCL2 family members. Mutations causing hearing loss or experimental truncation of the protein disrupt these interactions, releasing the amino terminal portion of the protein to initiate apoptosis by as yet unknown mechanisms. The varying effects of wild-type DFNA5 observed in the different cancer apoptosis studies may relate to levels of the protein expressed, and indicate a requirement for $\mathrm{p} 53$.

\section{CONCLUSIONS}

We have identified a number of potential BH3-only proteins which can now be evaluated by direct experimentation for BH3 domain-mediated interactions with BCL2 family members and regulation of cell death mechanisms. In addition to the candidates highlighted in the text, the list provided as Supplemental File $\mathbf{2}$ should prove to be a useful resource for further data mining of novel $\mathrm{BH} 3$-containing proteins. It is important to note, however, that this list is by no means a comprehensive compilation of all potential $\mathrm{BH} 3-$ only proteins in the human proteome. For example, the proapoptotic proteins BNIP1 and BNIP3 [89], which contain diverged BH3 motifs (Supplemental File 1), were not identified by this screen; nor were several other purported novel $\mathrm{BH} 3$-only proteins with atypical $\mathrm{BH} 3$-like sequences: such as MOAP1/MAP-1 (modulator of apoptosis) [90], SPIKE (small protein with inherent killing effect) [91], BLID/ BRCC2 (BH3-like motif containing, cell death inducer) [92], TGM2 (tissue transglutaminase) [93], APOL6 (apolipoprotein L6) [94], APOL1 (apolipoprotein L1) [95] or HEBP2/ SOUL [96]. Clearly, further relaxation in the stringency of the BH3 motif definition, and refinement of PSSM models and molecular dynamics simulations will be necessary to extend this strategy in order for it to become all inclusive.

We were struck by the fact that besides apoptosis the list of potential $\mathrm{BH} 3$-only candidates identified by the 13 -amino acid $\mathrm{BH} 3$ consensus sequence that we employed is enriched for proteins associated with autophagy (beyond BECN1) as well as a broad spectrum of cellular stress responses such as endoplasmic reticulum stress, oxidative stress, antiviral defense and the DNA damage response (Supplemental File 2). It seems possible that some of the $\mathrm{BH} 3$-like sequences, if not bona fide $\mathrm{BH} 3$ domains belonging to novel BCL2 family members, represent core modules that participate in proteinprotein interactions transmitting various stress signals to mitochondria and the nucleus, facilitating crosstalk between apoptotic pathways and those involved in autophagy and cell cycle checkpoint control [97-99]. In accord with this conjecture, during the writing of this manuscript Kimchi and colleagues published the results of a study in which they identified the autophagy protein ATG12 as a novel BH3- 


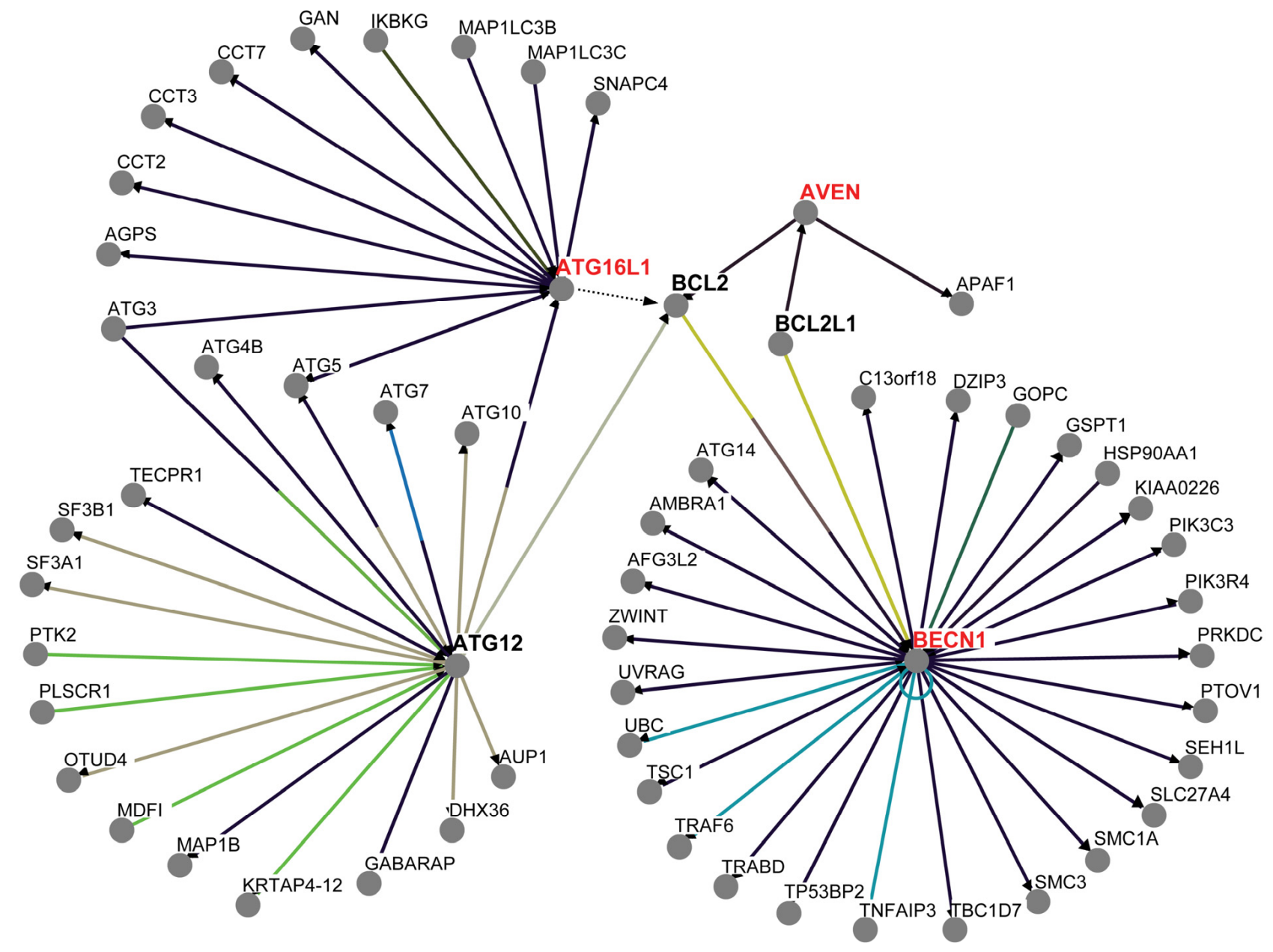

Fig. (4). Known and hypothetical interactions between selected autophagy, DNA damage checkpoint, and apoptotic proteins. Known physical associations for ATG12, ATG16L1, AVEN and BECN1 were imported from BioGrid (http://thebiogrid.org/). Binding of ATG12 to BCL2 via a BH3-like motif was reported in Ref. [100]. A putative interaction between ATG16L1 and a representative anti-apoptotic BCL2 family member (BCL2) is indicated. Proteins highlighted in red were recovered in the screen for new BH3-containing protein candidates.

only proapoptotic protein that binds to BCL2 and MCL-1 [100]. ATG12 is not present in our list of candidates because its atypical BH3-like region deviates from our consensus motif at two positions. However, one of the other autophagy genes that they identified as a positive regulator of apoptosis but did not characterize, ATG16L1, is present (Supplemental File 2, page 48) (Fig. 4).

\section{CONFLICT OF INTEREST}

None declared.

\section{ACKNOWLEDGEMENTS}

This work was supported in part by an American Cancer Society Pilot Grant for Junior Faculty from The George Washington University Cancer Institute IRG-08-091-01 and a Pilot Project Award from the Clinical and Translational Science Institute at Children's National Medical Center NIH Grant UL1RR031988 (I.R.); National Science Foundation Grant CDI-0941228 (C.Z.); and by NIH Grants R01HL65519 and R01HL66305, an Elaine H. Snyder
Cancer Research Award, a King Fahd Endowment and Dean's Funds from The George Washington University School of Medicine (R.G.H.). Its contents are solely the responsibility of the authors and do not necessarily represent the official views of the National Center for Research Resources or the National Institutes of Health.

\section{SUPPLEMENTARY MATERIAL}

Supplementary material is available on the publishers Web site along with the published article.

\section{REFERENCES}

[1] Shamas-Din A, Brahmbhatt H, Leber B, Andrews DW. BH3-only proteins: Orchestrators of apoptosis. Biochim Biophys Acta 2011; 1813: 508-20.

[2] Elkholi R, Floros KV, Chipuk JE. The role of BH3-only proteins in tumor cell development, signaling, and treatment. Genes Cancer 2011; 2: 523-37.

[3] Sattler M, Liang H, Nettesheim D, et al. Structure of Bcl-xL-Bak peptide complex: recognition between regulators of apoptosis. Science 1997; 275: 983-6. 
[4] Petros AM, Nettesheim DG, Wang Y, et al. Rationale for Bcl$\mathrm{xL} / \mathrm{Bad}$ peptide complex formation from structure, mutagenesis, and biophysical studies. Protein Sci 2000; 9: 2528-34.

[5] Lama D, Sankararamakrishnan R. Molecular dynamics simulations of pro-apoptotic BH3 peptide helices in aqueous medium: relationship between helix stability and their binding affinities to the anti-apoptotic protein Bcl-X(L). J Comput Aided Mol Des 2011; 25: 413-26.

[6] Day CL, Smits C, Fan FC, Lee EF, Fairlie WD, Hinds MG. Structure of the BH3 domains from the p53-inducible BH3-only proteins Noxa and Puma in complex with Mcl-1. J Mol Biol 2008; 380: $958-71$.

[7] Lanave C, Santamaria M, Saccone C. Comparative genomics: the evolutionary history of the Bcl-2 family. Gene 2004; 333: 71-9.

[8] Hinds MG, Day CL. Regulation of apoptosis: uncovering the binding determinants. Curr Opin Struct Biol 2005; 15: 690-9.

[9] Chen L, Willis SN, Wei A, et al. Differential targeting of prosurvival $\mathrm{Bcl}-2$ proteins by their $\mathrm{BH} 3$-only ligands allows complementary apoptotic function. Mol Cell 2005; 17: 393-403.

[10] Certo M, Del GMV, Nishino M, et al. Mitochondria primed by death signals determine cellular addiction to antiapoptotic BCL-2 family members. Cancer Cell 2006; 9: 351-65.

[11] Dutta S, Gulla S, Chen TS, Fire E, Grant RA, Keating AE. Determinants of BH3 binding specificity for Mcl-1 versus Bcl-xL. J Mol Biol 2010; 398: 747-62.

[12] Lee EF, Czabotar PE, Smith BJ, et al. Crystal structure of ABT737 complexed with Bcl-xL: implications for selectivity of antagonists of the Bcl-2 family. Cell Death Differ 2007; 14: 17113.

[13] Boersma MD, Sadowsky JD, Tomita YA, Gellman SH. Hydrophile scanning as a complement to alanine scanning for exploring and manipulating protein-protein recognition: application to the Bim BH3 domain. Protein Sci 2008; 17: 1232-40.

[14] Sabourin LA, Hawley RG. Suppression of programmed death and $\mathrm{G}_{1}$ arrest in B-cell hybridomas by interleukin-6 is not accompanied by altered expression of immediate early response genes. J Cell Physiol 1990; 145: 564-74.

[15] Schwarze MMK, Hawley RG. Prevention of myeloma cell apoptosis by ectopic $\mathrm{bcl}-2$ expression or interleukin-6-mediated upregulation of $b c l-x_{L}$. Cancer Res 1995; 55: 2262-5.

[16] Haughn L, Hawley RG, Morrison DK, von BH, Hockenbery DM. BCL-2 and BCL-XL restrict lineage choice during hematopoietic differentiation. J Biol Chem 2003; 278: 25158-65.

[17] Yang Z, Gagarin D, Ramezani A, Hawley RG, McCaffrey TA. Resistance to fas-induced apoptosis in cells from human atherosclerotic lesions: elevated Bcl-XL inhibits apoptosis and caspase activation. J Vasc Res 2007; 44: 483-94.

[18] Riz I, Zweier-Renn LA, Toma I, Hawley TS, Hawley RG. Apoptotic role of IKK in T-ALL therapeutic response. Mol Cancer Res 2011; 9: 979-84.

[19] Aurora R, Rose GD. Helix capping. Protein Sci 1998; 7: 21-38.

[20] Rost B, Yachdav G, Liu J. The PredictProtein server. Nucleic Acids Res 2004; 32: 321-6.

[21] Chen H, Van Duyne R, Zhang N, Kashanchi F, Zeng C. A novel binding pocket of cyclin-dependent kinase 2. Proteins 2009; 74: 122-32.

[22] van der Spoel D, Lindahl E, Hess B, Groenhof G, Mark AE, Berendsen HJ. GROMACS: fast, flexible, and free. J Comput Chem 2005; 26: 1701-18.

[23] Xiang Z, Honig B. Extending the accuracy limits of prediction for side-chain conformations. J Mol Biol 2001; 311: 421-30.

[24] Hess B, Bekker H, Berendsen HJC, Fraaije JGEM. LINCS: a linear constraint solver for molecular simulations. J Comput Chem 1997; 18: $1463-72$.

[25] Berendsen HJC, Postma JPM, van Gunsteren WF, Dinola A, Haak JR. Molecular-dynamics with coupling to an external bath. J Chem Phys 1984; 81: 3684-90.

[26] Chau BN, Cheng EH, Kerr DA, Hardwick JM. Aven, a novel inhibitor of caspase activation, binds Bcl-xL and Apaf-1. Mol Cell 2000; 6: 31-40.

[27] Kutuk O, Temel SG, Tolunay S, Basaga H. Aven blocks DNA damage-induced apoptosis by stabilising Bcl-xL. Eur J Cancer 2010; 46: 2494-505.

[28] Bratton SB, Salvesen GS. Regulation of the Apaf-1-caspase-9 apoptosome. J Cell Sci 2010; 123: 3209-14.
[29] Aouacheria A, Brunet F, Gouy M. Phylogenomics of life-or-death switches in multicellular animals: Bcl-2, BH3-Only, and BNip families of apoptotic regulators. Mol Biol Evol 2005; 22: 2395416.

[30] Chinnadurai G, Vijayalingam S, Rashmi R. BIK, the founding member of the BH3-only family proteins: mechanisms of cell death and role in cancer and pathogenic processes. Oncogene 2008; 27 (Suppl. 1): S20-9.

[31] Guo B, Godzik A, Reed JC. Bcl-G, a novel pro-apoptotic member of the Bcl-2 family. J Biol Chem 2001; 276: 2780-5.

[32] Moroy G, Martin E, Dejaegere A, Stote RH. Molecular basis for Bcl-2 homology 3 domain recognition in the Bcl-2 protein family: identification of conserved hot spot interactions. J Biol Chem 2009; 284: 17499-511.

[33] Ding J, Zhang Z, Roberts GJ, et al. Bcl-2 and Bax interact via the BH1-3 groove-BH3 motif interface and a novel interface involving the BH4 motif. J Biol Chem 2010; 285: 28749-63.

[34] Zou S, Chang J, LaFever L, et al. Identification of dAven, a Drosophila melanogaster ortholog of the cell cycle regulator Aven. Cell Cycle 2011; 10: 989-98.

[35] Lama D, Sankararamakrishnan R. Anti-apoptotic Bcl-XL protein in complex with BH3 peptides of pro-apoptotic Bak, Bad, and Bim proteins: comparative molecular dynamics simulations. Proteins 2008; 73: 492-514.

[36] Guo JY, Yamada A, Kajino T, et al. Aven-dependent activation of ATM following DNA damage. Curr Biol 2008; 18: 933-42.

[37] Gross A. A new Aven-ue to DNA-damage checkpoints. Trends Biochem Sci 2008; 33: 514-6.

[38] Komatsu K, Miyashita T, Hang H, et al. Human homologue of S. pombe Rad9 interacts with BCL-2/BCL-xL and promotes apoptosis. Nat Cell Biol 2000; 2: 1-6.

[39] Lee MW, Hirai I, Wang HG. Caspase-3-mediated cleavage of Rad9 during apoptosis. Oncogene 2003; 22: 6340-6.

[40] Meyerkord CL, Takahashi Y, Araya R, Takada N, Weiss RS, Wang HG. Loss of Hus1 sensitizes cells to etoposide-induced apoptosis by regulating BH3-only proteins. Oncogene 2008; 27: 7248-59.

[41] Zinkel SS, Hurov KE, Ong C, Abtahi FM, Gross A, Korsmeyer SJ. A role for proapoptotic BID in the DNA-damage response. Cell 2005; 122: 579-91.

[42] Kamer I, Sarig R, Zaltsman Y, et al. Proapoptotic BID is an ATM effector in the DNA-damage response. Cell 2005; 122: 593-603.

[43] Doerks T, Strauss M, Brendel M, Bork P. GRAM, a novel domain in glucosyltransferases, myotubularins and other putative membrane-associated proteins. Trends Biochem Sci 2000; 25: 4835.

[44] Stanelle J, Tu-Rapp H, Putzer BM. A novel mitochondrial protein DIP mediates E2F1-induced apoptosis independently of p53. Cell Death Differ 2005; 12: 347-57.

[45] John K, Alla V, Meier C, Putzer BM. GRAMD4 mimics p53 and mediates the apoptotic function of p73 at mitochondria. Cell Death Differ 2011; 18: 874-86.

[46] Hinds MG, Smits C, Fredericks-Short R, et al. Bim, Bad and Bmf: intrinsically unstructured $\mathrm{BH} 3$-only proteins that undergo a localized conformational change upon binding to prosurvival Bcl-2 targets. Cell Death Differ 2007; 14: 128-36.

[47] Gottfried I, Ehrlich M, Ashery U. The Sla2p/HIP1/HIP1R family: similar structure, similar function in endocytosis? Biochem Soc Trans 2010; 38: 187-91.

[48] Wanker EE, Rovira C, Scherzinger E, et al. HIP-I: a huntingtin interacting protein isolated by the yeast two-hybrid system. Hum Mol Genet 1997; 6: 487-95.

[49] Kalchman MA, Koide HB, McCutcheon K, et al. HIP1, a human homologue of $\mathrm{S}$. cerevisiae Sla2p, interacts with membraneassociated huntingtin in the brain. Nat Genet 1997; 16: 44-53.

[50] Seki N, Muramatsu M, Sugano S, et al. Cloning, expression analysis, and chromosomal localization of HIP1R, an isolog of huntingtin interacting protein (HIP1). J Hum Genet 1998; 43: 26871.

[51] Engqvist-Goldstein AE, Kessels MM, Chopra VS, Hayden MR, Drubin DG. An actin-binding protein of the Sla2/Huntingtin interacting protein 1 family is a novel component of clathrin-coated pits and vesicles. J Cell Biol 1999; 147: 1503-18.

[52] Hyun TS, Rao DS, Saint-Dic D, et al. HIP1 and HIP1r stabilize receptor tyrosine kinases and bind 3-phosphoinositides via epsin Nterminal homology domains. J Biol Chem 2004; 279: 14294-306. 
[53] Brett TJ, Legendre-Guillemin V, McPherson PS, Fremont DH. Structural definition of the F-actin-binding THATCH domain from HIP1R. Nat Struct Mol Biol 2006; 13: 121-30.

[54] Chopra VS, Metzler M, Rasper DM, et al. HIP12 is a nonproapoptotic member of a gene family including HIP1, an interacting protein with huntingtin. Mamm Genome 2000; 11: 1006-15.

[55] Rao DS, Bradley SV, Kumar PD, et al. Altered receptor trafficking in Huntingtin Interacting Protein 1-transformed cells. Cancer Cell 2003; 3: 471-82.

[56] Itoh T, Koshiba S, Kigawa T, Kikuchi A, Yokoyama S, Takenawa T. Role of the ENTH domain in phosphatidylinositol-4,5bisphosphate binding and endocytosis. Science 2001; 291: 104751

[57] Hackam AS, Yassa AS, Singaraja R, et al. Huntingtin interacting protein 1 induces apoptosis via a novel caspase-dependent death effector domain. J Biol Chem 2000; 275: 41299-308.

[58] Gervais FG, Singaraja R, Xanthoudakis S, et al. Recruitment and activation of caspase- 8 by the Huntingtin-interacting protein Hip-1 and a novel partner Hippi. Nat Cell Biol 2002; 4: 95-105.

[59] Rao DS, Hyun TS, Kumar PD, et al. Huntingtin-interacting protein 1 is overexpressed in prostate and colon cancer and is critical for cellular survival. J Clin Invest 2002; 110: 351-60.

[60] Rao DS, Chang JC, Kumar PD, et al. Huntingtin interacting protein 1 Is a clathrin coat binding protein required for differentiation of late spermatogenic progenitors. Mol Cell Biol 2001; 21: 7796-806.

[61] Kim JH, Yoon S, Won M, et al. HIP1R interacts with a member of Bcl-2 family, BCL2L10, and induces BAK-dependent cell death. Cell Physiol Biochem 2009; 23: 43-52.

[62] Ke H, Parron VI, Reece J, Zhang JY, Akiyama SK, French JE. BCL2 inhibits cell adhesion, spreading, and motility by enhancing actin polymerization. Cell Res 2010; 20: 458-69.

[63] Ke H, Zhang JY, Akiyama SK, French JE. BCL2 interaction with actin in vitro may inhibit cell motility by enhancing actin polymerization. Cell Adh Migr 2011; 5: 6-10

[64] Navarro-Lerida I, Martinez MM, Roncal F, Gavilanes F, Albar JP, Rodriguez-Crespo I. Proteomic identification of brain proteins that interact with dynein light chain LC8. Proteomics 2004; 4: 339-46.

[65] Puthalakath H, Huang DC, O'Reilly LA, King SM, Strasser A. The proapoptotic activity of the Bcl-2 family member Bim is regulated by interaction with the dynein motor complex. Mol Cell 1999; 3: 287-96.

[66] Day CL, Puthalakath H, Skea G, et al. Localization of dynein light chains 1 and 2 and their pro-apoptotic ligands. Biochem J 2004; 377: 597-605.

[67] Rapali P, Szenes A, Radnai L, Bakos A, Pal G, Nyitray L. DYNLL/LC8: a light chain subunit of the dynein motor complex and beyond. FEBS J 2011; 278: 2980-96.

[68] Steeg PS, Bevilacqua G, Kopper L, et al. Evidence for a novel gene associated with low tumor metastatic potential. J Natl Cancer Inst 1988; 80: 200-4

[69] Wallet V, Mutzel R, Troll H, et al. Dictyostelium nucleoside diphosphate kinase highly homologous to Nm23 and Awd proteins involved in mammalian tumor metastasis and Drosophila development. J Natl Cancer Inst 1990; 82: 1199-202.

[70] Boissan M, Dabernat S, Peuchant E, Schlattner U, Lascu I, Lacombe ML. The mammalian Nm23/NDPK family: from metastasis control to cilia movement. Mol Cell Biochem 2009; 329: 51-62.

[71] Desvignes T, Pontarotti P, Bobe J. Nme gene family evolutionary history reveals pre-metazoan origins and high conservation between humans and the sea anemone, Nematostella vectensis. PLoS One 2010; 5: e15506.

[72] Venturelli D, Martinez R, Melotti P, et al. Overexpression of DR$\mathrm{nm} 23$, a protein encoded by a member of the $\mathrm{nm} 23$ gene family, inhibits granulocyte differentiation and induces apoptosis in 32Dc13 myeloid cells. Proc Natl Acad Sci USA 1995; 92: 7435-9.

[73] Negroni A, Venturelli D, Tanno B, et al. Neuroblastoma specific effects of DR-nm23 and its mutant forms on differentiation and apoptosis. Cell Death Differ 2000; 7: 843-50.

[74] Kang Y, Lee DC, Han J, et al. NM23-H2 involves in negative regulation of Diva and Bcl2L10 in apoptosis signaling. Biochem Biophys Res Commun 2007; 359: 76-82.

[75] Song Q, Kuang Y, Dixit VM, Vincenz C. Boo, a novel negative regulator of cell death, interacts with Apaf-1. EMBO J 1999; 18: $167-78$.
[76] Lee R, Chen J, Matthews CP, McDougall JK, Neiman PE. Characterization of NR13-related human cell death regulator, Boo/Diva, in normal and cancer tissues. Biochim Biophys Acta 2001; 1520: 187-94.

[77] Venturelli D, Cesi V, Ransac S, Engelhard A, Perrotti D, Calabretta B. The nucleoside diphosphate kinase activity of DRnm23 is not required for inhibition of differentiation and induction of apoptosis in 32Dcl3 myeloid precursor cells. Exp Cell Res 2000; 257: 26571.

[78] Thompson DA, Weigel RJ. Characterization of a gene that is inversely correlated with estrogen receptor expression (ICERE-1) in breast carcinomas. Eur J Biochem 1998; 252: 169-77.

[79] Van Laer L, Huizing EH, Verstreken M, et al. Nonsyndromic hearing impairment is associated with a mutation in DFNA5. Nat Genet 1998; 20: 194-7.

[80] Akino K, Toyota M, Suzuki H, et al. Identification of DFNA5 as a target of epigenetic inactivation in gastric cancer. Cancer Sci 2007; 98: 88-95.

[81] Kim MS, Chang X, Yamashita K, et al. Aberrant promoter methylation and tumor suppressive activity of the DFNA5 gene in colorectal carcinoma. Oncogene 2008; 27: 3624-34.

[82] Kim MS, Lebron C, Nagpal JK, et al. Methylation of the DFNA5 increases risk of lymph node metastasis in human breast cancer. Biochem Biophys Res Commun 2008; 370: 38-43.

[83] Lage H, Helmbach H, Grottke C, Dietel M, Schadendorf D. DFNA5 (ICERE-1) contributes to acquired etoposide resistance in melanoma cells. FEBS Lett 2001; 494: 54-9.

[84] Masuda Y, Futamura M, Kamino H, et al. The potential role of DFNA5, a hearing impairment gene, in p53-mediated cellular response to DNA damage. J Hum Genet 2006; 51: 652-64.

[85] Webb MS, Miller AL, Thompson EB. In CEM cells the autosomal deafness gene dfna5 is regulated by glucocorticoids and forskolin. J Steroid Biochem Mol Biol 2007; 107: 15-21.

[86] Park HJ, Cho HJ, Baek JI, et al. Evidence for a founder mutation causing DFNA5 hearing loss in East Asians. J Hum Genet 2010; 55: 59-62.

[87] Van Laer L, Vrijens K, Thys S, et al. DFNA5: hearing impairment exon instead of hearing impairment gene? J Med Genet 2004; 41 401-6.

[88] Op de Beeck K, Van Camp G, Thys S, et al. The DFNA5 gene, responsible for hearing loss and involved in cancer, encodes a novel apoptosis-inducing protein. Eur J Hum Genet 2011; 19: 965 73.

[89] Zhang J, Ney PA. Role of BNIP3 and NIX in cell death, autophagy, and mitophagy. Cell Death Differ 2009; 16: 939-46.

[90] Tan KO, Tan KM, Chan SL, et al. MAP-1, a novel proapoptotic protein containing a $\mathrm{BH} 3$-like motif that associates with Bax through its Bcl-2 homology domains. J Biol Chem 2001; 276: 2802-7.

[91] Mund T, Gewies A, Schoenfeld N, Bauer MK, Grimm S. Spike, a novel $\mathrm{BH} 3$-only protein, regulates apoptosis at the endoplasmic reticulum. FASEB J 2003; 17: 696-8.

[92] Broustas CG, Gokhale PC, Rahman A, Dritschilo A, Ahmad I, Kasid U. BRCC2, a novel BH3-like domain-containing protein, induces apoptosis in a caspase-dependent manner. J Biol Chem 2004; 279: 26780-8.

[93] Rodolfo C, Mormone E, Matarrese $\mathrm{P}$, et al. Tissue transglutaminase is a multifunctional $\mathrm{BH} 3$-only protein. J Biol Chem 2004; 279: 54783-92.

[94] Liu Z, Lu H, Jiang Z, Pastuszyn A, Hu CA. Apolipoprotein 16, a novel proapoptotic $\mathrm{Bcl}-2$ homology 3 -only protein, induces mitochondria-mediated apoptosis in cancer cells. Mol Cancer Res 2005; 3: 21-31.

[95] Wan G, Zhaorigetu S, Liu Z, Kaini R, Jiang Z, Hu CA. Apolipoprotein L1, a novel Bcl-2 homology domain 3-only lipidbinding protein, induces autophagic cell death. J Biol Chem 2008; 283: 21540-9.

[96] Szigeti A, Hocsak E, Rapolti E, et al. Facilitation of mitochondrial outer and inner membrane permeabilization and cell death in oxidative stress by a novel Bcl-2 homology 3 domain protein. $\mathrm{J}$ Biol Chem 2010; 285: 2140-51.

[97] Behrends C, Sowa ME, Gygi SP, Harper JW. Network organization of the human autophagy system. Nature 2010; 466: 68-76.

[98] Danial NN, Gimenez-Cassina A, Tondera D. Homeostatic functions of BCL-2 proteins beyond apoptosis. Adv Exp Med Biol 2010; 687: 1-32. 
[99] Tabas I, Ron D. Integrating the mechanisms of apoptosis induced by endoplasmic reticulum stress. Nat Cell Biol 2011; 13: 184-90.

[100] Rubinstein AD, Eisenstein M, Ber Y, Bialik S, Kimchi A. The autophagy protein Atg12 associates with antiapoptotic Bcl-2 family members to promote mitochondrial apoptosis. Mol Cell 2011; 44: 698-709.

Received: February 17, 2012

Revised: March 30, 2012

Accepted: April 02, 2012

(C) Hawley et al.; Licensee Bentham Open.

This is an open access article licensed under the terms of the Creative Commons Attribution Non-Commercial License (http://creativecommons.org/licenses/by$\mathrm{nc} / 3.0 /$ ), which permits unrestricted, non-commercial use, distribution and reproduction in any medium, provided the work is properly cited. 